\title{
Human Modeling For Ground Processing Human Factors Engineering Analysis
}

Lecture

\section{Abstract}

There have been many advancements and accomplishments over that last few years using human modeling for human factors engineering analysis for design of spacecraft and launch vehicles. The key methods used for this are motion capture and computer generated human models. The focus of this paper is to explain the different types of human modeling used currently and in the past at Kennedy Space Center (KSC) currently, and to explain the future plans for human modeling for future spacecraft designs.

\section{Summary}

The purpose of this study is to describe the different human modeling used at the Kennedy Space Center for human factor analysis. The work in this proposal includes literature review of work performed at KSC and discussions with experts in the field of human modeling at the Kennedy Space Center. As will be shown in this work, there have been several successes by using human modeling for analyzing the human activities in spacecraft processing. We are now at the point where the knowledge, skills and experience from the past can be combined with the current direction for technology to enable even greater strides to be made for human modeling for human factors analysis for future space flight systems.

Motion Capture modeling

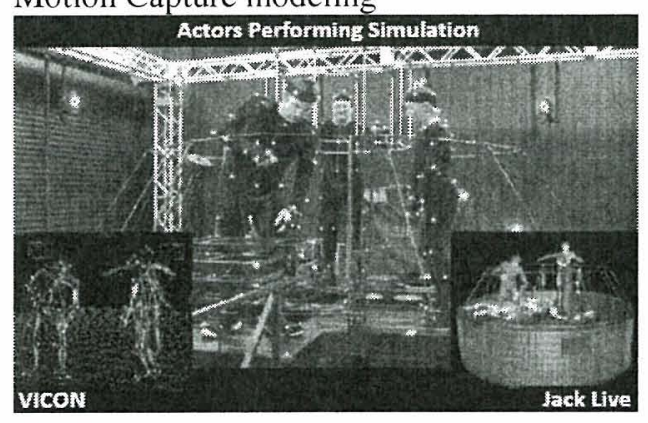

Computer Models

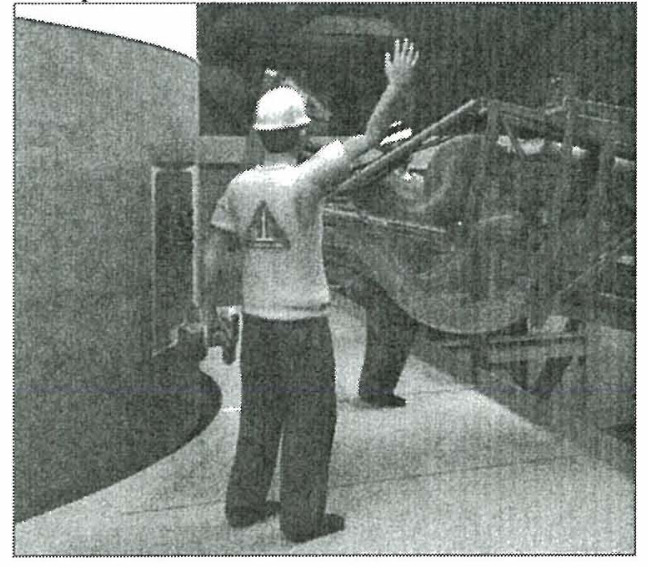

